Edited by Kiriakos Xenitidis and
Colin Campbell
Contents
- Confectionery consumption and violence
- Structural brain abnormalities in bipolar disorder
- Evolution and psychiatry

\section{Confectionery consumption and violence}

Moore et al ${ }^{1}$ found a 'novel and robust' relationship between confectionery consumption during childhood and conviction for violence in adulthood. However, there are serious methodological concerns, which make the overall findings questionable. As the authors recognise, the number of violent people in their cohort is very low. The lack of descriptive information in the paper, contrary to recommendations on the reporting of observational studies, ${ }^{2}$ forces the reader to an exercise of reconstruction. What emerges is that only about 33 participants were violent $(0.47 \%$ of 6942 ) and of these, only 23 (69\% of the 33 violent individuals) had eaten confectionery excessively. With such numbers, it is highly discouraged in the biostatistical literature to model the probability of being violent using as many parameters (8) as the authors did, since the fit is essentially driven by the number of cases and not by the entire sample size. ${ }^{3}$ The deficiencies of this approach are well known and numerous, affecting all aspects of the modelling process, from variable selection to effect size estimation, ${ }^{4}$ and are not, generally, accommodated by the adoption of rare-events logistical models, which only provide a fix for bias in estimating regression parameters. With such few cases, no interactions have been considered, even though some may be very intuitive (e.g. confectionery consumption and child-oriented parenting). With no serious attempt at considering interactions in the model, the risk of finding spurious associations is well documented (Simpson's paradox). ${ }^{5}$ Unfortunately, no details are provided in the paper concerning distribution of the other seven factors included in the multivariable model (gender, late birth, etc.) between violent and non-violent people, so that it is almost impossible to understand how low the cell frequency is in some such combinations. With these considerations in mind, the conclusions suggesting a relationship between confectionery and violence seem an over-interpretation of the fitted model.

1 Moore SC, Carter LM, van Goozen SHM. Confectionery consumption in childhood and adult violence. Br J Psychiatry 2009; 195: 366-7.

2 Vandenbroucke JP, von Elm E, Altman DG, Gotzsche PC, Mulrow CD, Pocock $\mathrm{SJ}$, et al. Strengthening the Reporting of Observational Studies in Epidemiology (STROBE): explanation and elaboration. Epidemiology 2007; 18: $805-35$.

3 Harrell FE Jr, Lee KL, Mark DB. Multivariable prognostic models: issues in developing models, evaluating assumptions and adequacy, and measuring and reducing errors. Stat Med 1996; 15: 361-87.

4 Harrell FE Jr, Lee KL, Califf RM, Pryor DB, Rosati RA. Regression modelling strategies for improved prognostic prediction. Stat Med 1984; 3: 143-52.

5 Agresti A. Categorical Data Analysis (2nd edn). Wiley \& Sons, 2002.

D. Gregori, Department of Environmental Medicine and Public Health, University of Padova, Italy. Email: dario.gregori@unipd.it

doi: 10.1192/bjp.196.3.245
Author's reply: We take issue with Gregori's statement that methodological concerns render the overall findings of our report ${ }^{1}$ questionable. Gregori correctly observes that 'the number of violent people in their cohort is very low' and goes on to suggest that reporting results on such small samples should be discouraged. We are interested in life-course factors that predict adult violence in the hope that such research might inform early life-course interventions. We therefore have two options. Either recruit violent offenders and enquire of their childhoods, or follow a cohort of individuals recording information on their circumstances to assess associations with later problem behaviour. Unfortunately, compounding the vagaries of human memory are the particular difficulties many offenders have with recalling what they did the previous day, let alone several decades ago. It is thus unfeasible to conduct retrospective studies; this leaves cohort studies as the only realistic and robust methodology. We are fortunate in the UK to have one of the most highly regarded cohort studies in the world, but despite its large initial sample size the rarity of violence means that only a small number of respondents demonstrate the behaviour of interest. Should we, as Gregori counsels, simply not consider using the British Cohort Study to look into childhood factors predicting adult violence because violence is rare? We suggest that this would be a valuable and informative resource squandered if that advice were followed. Gregori also suggests that models on rare data should not involve too many covariates. In our short paper we reported that we considered various configurations including the unadjusted association between confectionery and violence and that the strength of association was consistent across models - analytically we did as much as we could to test this association. We chose not to report simpler models and hardly mentioned the extensive analyses assessing the impact of attrition simply because we felt this paper suited a short-report format and including this additional information would only detract from what was a perfectly well-articulated finding. We therefore maintain that we analysed some of the best cohort data available to assess childhood predictors of an important outcome and found a robust association. We were honest with regard to the sample size, concluding in the paper that this is one area that should be addressed before firm conclusions can be drawn.

1 Moore SC, Carter L, Van Goozen SHM. Confectionery consumption in childhood and adult violence. Br J Psychiatry 2009; 195: 366-7.

Simon C. Moore, Violence and Society Research Group, Applied Clinical Research and Public Health, School of Dentistry, University of Cardiff, Cardiff CF14 4XY, UK. Email: mooresc2@cardiff.ac.uk

doi: $10.1192 / \mathrm{bjp} .196 .3 .245 a$

\section{Structural brain abnormalities in bipolar disorder: what meta-analyses tell us}

Findings from Arnone et al's ${ }^{1}$ systematic review and meta-analysis of magnetic resonance imaging (MRI) studies suggest that patients with bipolar disorder are characterised, in comparison with healthy controls, by significant reductions of whole-brain and prefrontal lobe volumes and by enlargement of lateral ventricles and globus pallidus, although most of the brain changes detected in bipolar disorder do not seem to be diagnostically specific and some clinical variables, such as patients' age, duration of illness and pharmacological treatment, appear to be relevant in determining the magnitude of observed effect sizes.

These findings are not completely consistent our own recent meta-analysis ${ }^{2}$ of MRI studies in first-episode bipolar disorder. 
Our study did not evidence whole-brain volume deficits in firstepisode bipolar disorder compared with healthy controls. This may indicate that a progressive decrease of whole-brain volume occurs over the course of the disease, and might be detectable only when multi-episode or chronic cases are considered. This is confirmed by the correlation found between gray matter loss and duration of illness in the meta-regression performed by Arnone et $a l^{1}$ and by the results of longitudinal studies demonstrating gray matter volume loss over time in the prefrontal cortex in young adults with bipolar disorder ${ }^{3}$ or of cross-sectional comparisons between first- and multiple-episode bipolar disorder showing more severe brain abnormalities in patients with multiple episodes of illness. ${ }^{4}$

On the other hand, we did find a significant decrease of total white matter volume in first-episode bipolar disorder, while Arnone et $a l^{1}$ failed to obtain the same finding in their analysis of a larger number of studies mainly conducted in patients with chronic illness. This may indicate that alterations in white matter normal growth may constitute early and primary abnormalities in bipolar disorder, consistent with some preliminary evidence of the association between patterns of disturbed structural white matter integrity in bipolar disorder and genetic liability for the illness. ${ }^{5}$ In order to explain the lack of white matter volume reduction in chronic illness, it could be hypothesised either that other, more generalised brain changes may override white matter abnormalities over the course of the disease, or that white matter changes may be attenuated by treatment or, again, may be less sensitive to the later effects of ageing. Indirect support for this idea derives from the finding of smaller volumetric differences in the temporal lobes in bipolar disorder with increasing age, duration of illness and use of mood stabilisers, ${ }^{1}$ the only discrete brain volume including white matter analysed in the meta-regressions performed by Arnone et al.

In conclusion, the finding of different brain abnormalities in chronic $v$. first-episode bipolar disorder supports the notion of different pathophysiological trajectories of specific brain morphological characteristics over the course of the disease and emphasises the need for further longitudinal studies aimed at addressing specifically the issue of the time of appearance and course of individual brain abnormalities in bipolar disorder, from which may derive a better understanding of the pathogenesis of the disease itself.

1 Arnone D, Cavanagh J, Gerber D, Lawrie SM, Ebmeier KP, McIntosh AM. Magnetic resonance imaging studies in bipolar disorder and schizophrenia: meta-analysis. Br J Psychiatry 2009; 195: 194-201.

2 Vita A, De Peri L, Sacchetti E. Gray matter, white matter, brain, and intracranial volumes in first-episode bipolar disorder: a meta-analysis of magnetic resonance imaging studies. Bipolar Disord 2009; 11: 807-14.

3 Kalmar JH, Wang $\mathrm{F}$, Spencer L, Edmiston $\mathrm{E}$, Lacadie $\mathrm{CM}$, Martin A, et al. Preliminary evidence for progressive prefrontal abnormalities in adolescents and young adults with bipolar disorder. J Int Neuropsychol Soc 2009; 15 476-81.

4 Strakowski SM, DelBello MP, Zimmerman ME, Getz GE, Mills NP, Ret J, et al. Ventricular and periventricular structural volumes in first versus multipleepisode bipolar disorder. Am J Psychiatry 2002; 159: 1841-7.

5 Chaddock CA, Barker GJ, Marshall N, schulze $\mathrm{K}$, Hall MH, et al. White matte microstructural impairments and genetic liability to familial bipolar I disorder. Br J Psychiatry 2009; 194: 527-34.

Antonio Vita, Luca De Peri, Emilio Sacchetti, University of Brescia, School of Medicine, Deparment of Mental Health, Spedali Civili Hospital, Brescia, Italy. Email: vita@med.unibs.it

doi: 10.1192/bjp.196.3.245b

Author's reply: We are grateful to Drs Vita, Peri and Sacchetti, who raise the very important point that morphometric abnormalities detectable in first-onset bipolar disorder appear different from those described in chronic patients. An observation which, as Vita et al suggest, may underpin important information about the pathogenesis of the disorder and would benefit from clarification emerging from longitudinal studies. Prompted by their meta-analysis ${ }^{1}$ and our own work, ${ }^{2}$ we have conducted further analyses by including only patients with first-episode bipolar disorder $v$. healthy controls. Despite methodological differences and different inclusion and exclusion criteria, we are in agreement with Vita et al. We found no evidence of whole-brain volume reduction in the first-episode patients $v$. healthy controls (effect size -0.23 ; $95 \%$ CI -0.47 to $0.002 ; I^{2}=0, P=0.51$; Egger's $P=0.31$ ). This finding supports Vita et als hypothesis that wholebrain volume loss may be occurring with illness progression and/ or its epiphenomena (e.g. number of episodes, pharmacological treatment). Similarly we found no evidence of gray matter loss (effect size $-0.02 ; 95 \%$ CI -0.40 to $0.37 ; I^{2}=0.02, P=0.36$; Egger's $P=0.16)$ but significant white matter volumetric reduction in the first-episode patients $v$. healthy controls (effect size $-0.45 ; 95 \% \mathrm{CI}-0.85$ to $-0.06 ; I^{2}=0.04, P=0.35$; Egger's $P=0.68$ ). These and other observations ${ }^{3,4}$ support the possibility that white matter deficits have a particular relevance to the aetiology of bipolar disorder. However, the paucity of first-episode studies is reflected in the relatively wide confidence intervals around our estimates. Further studies of patients with firstepisode bipolar disorder, as well as cohort and high-risk studies, are necessary if we are to improve our understanding of the role of structural changes in the pathogenesis of this condition.

1 Vita A, De Peri L, Sacchetti E. Gray matter, white matter, brain, and intracranial volumes in first-episode bipolar disorder: a meta-analysis of magnetic resonance imaging studies. Bipolar Disord 2009; 11: 807-14.

2 Arnone D, Cavanagh J, Gerber D, Lawrie SM, Ebmeier KP, McIntosh AM Magnetic resonance imaging studies in bipolar disorder and schizophrenia: meta-analysis. Br J Psychiatry 2009; 195: 194-201.

3 Sussmann JE, Lymer GK, McKirdy J, Moorhead TW, Maniega SM, Job D, et al. White matter abnormalities in bipolar disorder and schizophrenia detected using diffusion tensor magnetic resonance imaging. Bipolar Disord 2009; 11: $11-8$

4 McIntosh AM, Maniega SM, Lymer GK, McKirdy J, Hall J, Sussmann JE, et al. White matter tractography in bipolar disorder and schizophrenia. Biol Psychiatry 2008; 64: 1088-92.

D. Arnone, Neuroscience and Psychiatry Unit, University of Manchester, G810 Stopford Building, Oxford Road, Manchester M13 9PT, UK.

Email: danilo.arnone@manchester.ac.uk

doi: 10.1192/bjp.196.3.246

\section{Evolution and psychiatry}

If evolution is the missing half of a 'truly biological psychiatry', the other half being biological reductionism, then value is out of the picture. But this cannot be. We do not deny the gains from biology or those that are to come (millions of people manage to live because of advances in this field). Nor are we pessimistic about the potential gains that evolution claims for mental healthcare. However, these two 'halves' do not make a whole. We understand the aspiration for a truly biological psychiatry: life would be easier. Biology (although a big part, or the major part of the picture) cannot (alas!) be the whole, and evolutionary theoretical considerations of disorder, natural function, design and the like cannot fill what is missing. The reason is that even if we accept a value-free account of naturally selected mechanisms, physical as well as mental, these must be considered within the spectrum of individual and social values. Fulford ${ }^{2}$ explains why values are so feared. Other theorists who have considered 